
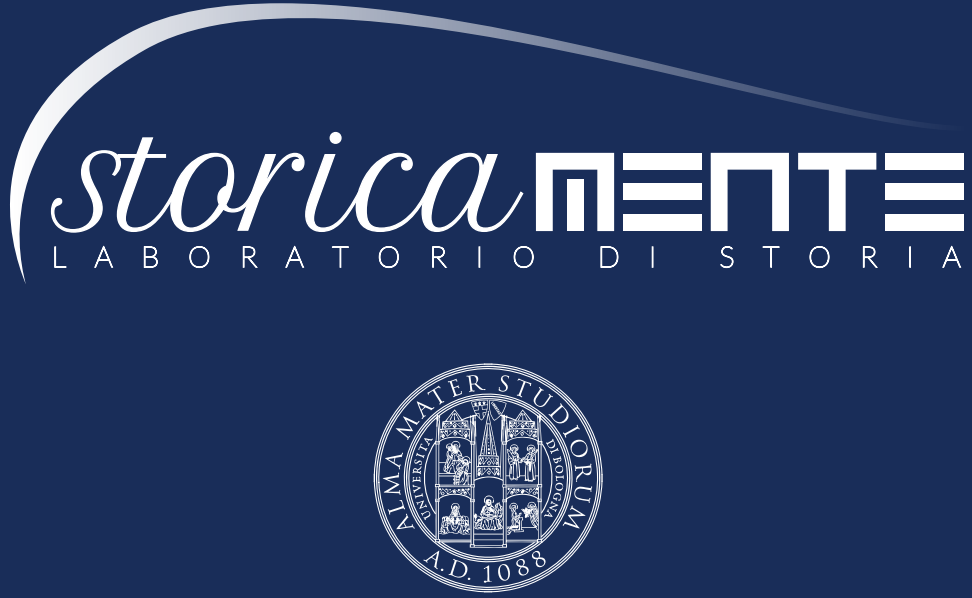

ALMA MATER STUDIORUM

Università di Bologna

Dipartimento di Storia Culture Civiltà

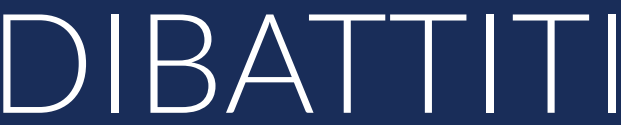

INTERVISTA

VIELLA 
STORICAMENTE.ORG

Laboratorio di Storia

Gabriella Gribaudi

La memoria delle catastrofi nei processi di ripartenza. Una conversazione

(C) Gabriella Gribaudi

Creative Commons BY-NC-ND 4.0

International License 2004- 2021

Volume: 2021

Issue: 17

Article number 8

Section: Dibattiti

Pages. 1-25

DOI: $10.52056 / 9788833138732 / 08$

ISSN: $1825-411 \mathrm{X}$

Publisher: Viella

Double blind peer review: No

Document type: Editorial Material

Research Areas: History

Published: 14/12/2021

Corresponding Address: Gabriella Gribaudi, Università di Napoli Federico II, Dipartimento di Scienze Sociali, Vico Monte della Pietà 1, 80138 Napoli, Italy 


\title{
La memoria delle catastrofi nei processi di ripartenza. Una conversazione
}

\author{
Gabriella Gribaudi \\ Univ. di Napoli Federico II, Dipartimento di Scienze Sociali \\ gabri.gribaudi@gmail.com \\ intervista a cura di MARICA TOLOMELLI ${ }^{\star}$
}

The Memory of Catastrophes in the Processes of Restarting. A Conversation

Events, which like bombardments, massacres as well as "natural" disasters provoke the sudden, often unexpected destruction of living conditions for individuals and communities, are traumatic experiences whose effects can last for generations. Both the memory and the history of such traumatic experiences in 20th century's Europe - bombardments during WWII and earthquakes until the 1980s - how they were perceived by the affected people and the reactions they unleashed are the subject of Gabriella Gribaudi's recent study, La memoria, i traumi, la storia. La guerra e le catastrofi del Novecento (Viella 2020). How to resume after having lost everything, what individual and collective strategies do people adopt to create a new context of ordinary daily life, how to manage the tension between the wish to recreate the lost conditions and the need to shape a new prospect of life are the questions discussed with the author in the following interview.

KEYWORDS: HISTORY, MEMORIES, NATURAL CATASTROPHES, SECOND WORLD WAR, SHOAH, EARTHQUAKES

Conosciamo e apprezziamo la storica Gabriella Gribaudi per l'attenzione che da anni dedica agli effetti prodotti da esperienze traumatiche, guerre in particolare, su soggetti e collettività. Subire violenze, trovarsi a reagire di fronte a catastrofi causa di perdite incommensurabili, come la perdita dei propri cari, della casa, dei propri averi o dell'ambiente della vita quotidiana, esige enormi investimenti in termini di risorse - emotive e psicologiche, ma anche materiali e sociali. Uno sforzo di risollevamento e ripresa che può indurre in un primo momento a rimuovere il trauma subito per sopravvivere e proiettarsi sul futuro; tuttavia, nel più lungo periodo le esperienze di eventi traumatici e le modalità del loro superamento divengono oggetto di rielaborazioni più o meno consapevoli attraverso il forgiarsi di molteplici stratificazioni memoniche: memorie pubbliche e memorie private, di singoli individui e di collettività, memorie consensuali e memorie confliggenti... Di questi temi Gribaudi si è occupata in suoi numerosi studi, tra cui si ricorda in particolare il brillante volume - Guerra totale. Tra bombe

^Università di Bologna, Dipartimento di Storia Culture e Civiltà; marica.tolomelli@unibo.it. 
alleate e violenze naziste. Napoli e il fronte meridionale 1940-44 (2005) - sulla particolare esperienza della Seconda guerra mondiale in Sud Italia, dove la popolazione si è trovata a subire le violenze, i bombardamenti, gli effetti catastrofici della guerra di occupazione tedesca e di quella di liberazione alleata, arricchendo con un contributo importante il vasto ambito storiografico che negli ultimi decenni si è affermato nell'incrocio tra studi sui traumi e studi sulla memoria.

Rispetto ai suoi precedenti lavori e marcando anche una significativa distinzione rispetto agli approcci e agli interessi di ricerca dominanti la storiografia sulle memorie traumatiche, il recente volume di Gribaudi, La memoria, $i$ traumi, la storia. La guerra e le catastrofi nel Novecento (2020), presenta un importante elemento di novità: l'accostamento di memorie sviluppatesi attorno a traumi prodotti da catastrof "naturali" - terremoti in primis - a quelle relative a traumi prodotti dalla Seconda guerra mondiale in Italia e in Europa. Come in più passaggi sottolinea l'autrice, è evidente che si tratti di eventi estremamente distanti sotto il profilo del coinvolgimento umano e delle implicazioni politiche: imprevedibilità della natura nel primo caso, causa dell'azione e della volontà umana di infierire e produrre danni nel secondo. Ma a parte il fatto che anche gli effetti delle catastrofi naturali possono essere messi in conto se non previsti e per questo contenuti - basti evocare il caso esemplare di come il Giappone abbia sviluppato una politica di "governo" dei terremoti - ciò che in parte accomuna i traumi prodotti dalla Seconda guerra mondiale a quelli di causa naturale è la percezione delle persone, lo shock e i sentimenti di disorientamento suscitati dall'irruzione - improvvisa e violenta - di eventi catastrofici. Avvalendosi di fonti orali - sul cui impiego Gabriella Gribaudi ha sviluppato una notevole expertise - l'autrice rileva come nella memoria soggettiva gli effetti prodotti da bombardamenti o terremoti siano ricordati soprattutto per la loro potenza distruttrice, ponendo in secondo piano contesto storico e responsabilità. Al punto da riscontrare le somiglianze tra "le descrizioni degli abitanti di Amburgo, distrutta dalle bombe nel 1943, e quelle degli abitanti di Laviano, paese dell'Irpinia raso al suolo dal terremoto nel 1980, in un certo senso atemporali e astoriche" (9). Spetta certamente al lavoro storiografico la precisa collocazione di esperienze e memorie nei rispettivi contesti storici, compito che l'autrice assolve pienamente, ma il dato interessante e che legittima l'analisi quasi comparativa di memorie traumatiche legate a eventi profondamente eterogenei risulta convincente soprattutto per la prospettiva analitica seguita.

Nell'ambito del dossier di Storicamente dedicato al tema delle ripartenze a seguito di eventi catastrofici - un dossier da cui volutamente si sono escluse le guerre non solo per non ampliare l'orizzonte a un ambito di ricerca già ampiamente battuto, ma proprio per riportare l'attenzione su tipologie di catastrofi di cui, come la stessa Gribaudi osserva, la storiografia si è occupata in 
misura minore - la redazione ha pensato di intervistare Gabriella Gribaudi in merito all'ultima parte del suo libro, quella dedicata alla memoria delle catastrofi "naturali" (217-67). Proponiamo di seguito l'intervista.

Puoi precisare l'uso del termine catastrofe in ambito storiografico e nel tuo libro in particolare?

La parola stessa "catastrofe" rimanda al concetto di sconvolgimento, rovesciamento.

La catastrofe, è stato detto, è un avvenimento che rompe il corso ordinario delle cose, interrompe il tempo umano, apre un baratro tra passato e presente. Costituisce una minaccia radicale all'ordine culturale e sociale nella sua dimensione spaziale e nella sua dimensione temporale. Si tratta di una categoria polisemica che può riguardare fatti naturali, politici, sociali. Proprio per il suo carattere evenemenziale e dirompente è stata rappresentata spesso come un evento fuori della storia, un evento atemporale. I disastri "naturali", considerati incidenti al di fuori del controllo o dell'azione umana, sono entrati con difficoltà nell'alveo degli studi storici. La storia, tuttavia, può dare e ha dato in alcuni casi un contributo importante per declinare le categorie e le interpretazioni delle catastrofi nei diversi contesti storici e materiali.

Nella società tradizionale, dal medioevo alla prima età moderna, gli eventi catastrofici erano definiti flagelli inviati da dio per correggere, ammonire, castigare gli uomini per le loro trasgressioni e i loro peccati. Venivano rappresentati con immagini bibliche: il giorno del giudizio, l'apocalisse... E la difesa ufficiale erano la preghiera e il culto dei santi: cappelle votive, statue di santi protettori venivano collocate in punti strategici per proteggere le persone dai pericoli (san Gennaro a Napoli che alza la mano contro la lava del Vesuvio, san Giovanni Nepomuceno sui fiumi...). Rituali religiosi e processioni percorrevano il territorio colpito dalla catastrofe. Con il terremoto di Lisbona del 1755, cui fa seguito la discussione degli illuministi sul ruolo della natura e dell'azione umana, l'interpretazione della catastrofe viene desacralizzata e l'attenzione si sposta sul tema del rischio e sull'azione degli uomini per prevenirlo. Viene posto l'accento sul protagonismo dell'uomo e sulla sua capacità di dominare la natura. 
Il progresso è la nuova dimensione teleologica: come scrive Walter, la divina provvidenza viene sostituita dal progresso provvidenziale. Lo sviluppo del progresso tecnologico provoca poi paradossalmente nuove incertezze e vulnerabilità, moltiplicando le cosiddette catastrofi antropiche completamente generate dalla mano umana.

Gli storici fanno notare come questo cammino, da un approccio irrazionale ai disastri caratteristico delle società antiche a una gestione razionale e scientifica caratteristica delle società moderne, sia in realtà una ricostruzione ideologica e semplificata di una storia ben più complessa: chi portava i santi in processione poteva nello stesso tempo cercare le soluzioni materiali per prevenire il rischio, come hanno mostrato ad esempio Christian Pfister che ha studiato la prevenzione delle inondazioni dei fiumi e delle valanghe da parte delle popolazioni alpine a partire dal Quattrocento e Greg Bankoff che ha analizzato la "cultura del disastro" nelle Filippine dalla colonizzazione spagnola fino ai nostri giorni.

D'altro canto la tecnologia e il discorso scientifico non sempre sono in grado in grado di costituire "una risposta sufficiente alle angosce quotidiane delle popolazioni. La spiegazione razionale non elude la necessità di continuare a convivere con le minacce" (Walter 2009) e fare ricorso quindi, insieme alla fiducia nell'intervento scientifico e tecnico, a riti e linguaggi sacrali per scongiurare il pericolo. La lettura razionale e quella religiosa coabitano nella lunga durata dal XVI al XX secolo: devozioni, superstizioni, fatalismo convivono con la richiesta di protezione tecnologica e di previsione scientifica.

La percezione del rischio, le paure, le interpretazioni delle catastrofi vanno viste all'interno dei contesti storici, materiali e culturali in cui le popolazioni vivono. L'approccio storico è fondamentale per inscrivere l'evento catastrofico nella dimensione del tempo. Per capire le trasformazioni della natura come dell'intervento umano è necessario introdurre la diacronia; gli storici tuttavia, tranne poche eccezioni, si sono raramente misurati sul tema. In Italia in particolare, la storia delle catastrofi e dei processi di ricostruzione non è entrata nel mainstream della storiografia contemporanea, che è stata e continua a essere ancora eminentemente politica. Sfido chiunque a trovare nei volumi dedicati alla storia dell'Italia repubblicana notizie sui tanti disastri che hanno colpito il nostro paese. Se ci sono è solo, come per il caso del terremo- 
to campano-lucano del 1980, per mostrarne i risvolti politici a livello nazionale.

Il ruolo degli storici ha invece avuto una funzione preminente nello studio di quelle che nel corso del Novecento fino ad oggi sono state collocate nella categoria di catastrofi: le guerre e i genocidi. La stessa parola "Shoah" significa catastrofe e a catastrofe corrisponde il termine speculare di "Nakba", utilizzato dai palestinesi per indicare la sconfitta e la fuga dalla Palestina. Si tratta di mondi perduti senza speranza, e, per tornare all'etimologia della parola, di veri e propri sconvolgimenti nelle vite e nelle geografie politiche e materiali dei territori. Nasce così un filone di studi che prende significativamente il nome di "trauma studies", che si incrocia e si confonde con il vero e proprio boom costituito, a partire dagli anni Novanta del secolo scorso, dai "memory studies" incentrati sulla dimensione soggettiva del trauma e sulla testimonianza di vittime e sopravvissuti.

Pensi sia necessario ridefinire il concetto di catastrofi in relazione a quello di antropocene? Potrebbe essere stimolante questo ripensamento per l'indagine storiografica?

A cominciare dal noto volume di Henry Quarantelli What is a Disaster? pubblicato nel 1998, in tutti i lavori, pur con differenti declinazioni e metodologie, il disastro viene presentato innanzitutto come un costrutto sociale e non un evento immutabile e fisso. L'evento catastrofico è la conseguenza di un'interazione fra natura e intervento umano. L'esempio concreto può essere costituito dai terremoti: i terremoti sono stati definiti "unnatural disasters" (Hewitt 1997), proprio perché la misura della catastrofe in termini di vittime e di devastazioni è prodotta dall'intervento umano e dalla scarsa prevenzione attuata negli anni precedenti l'evento sismico. Quando si verifica un terremoto, si scopre spesso che il luogo distrutto - più volte nella storia - non era catalogato tra i territori a maggior rischio sismico. È successo in Irpinia, dove Sant'Angelo dei Lombardi e Lioni, due delle cittadine che hanno subito i maggiori danni con il crollo degli edifici più moderni e recenti, fra cui l'ospedale, costruito pochi anni prima, non erano classificate come a elevato pericolo sismico. Ma in Italia gli esempi potrebbero essere infiniti.

Se le catastrofi naturali sono spesso amplificate dall'intervento umano, esiste una serie di calamità e disastri interamente dovuti all'azione 
dell'uomo e causati proprio da quel progresso tecnologico, che in una visione razionalista era stato visto come la soluzione definitiva nella lotta contro i pericoli insiti nella natura. Le catastrofi antropiche sembrano segnare la storia della contemporaneità producendo nuove incertezze, nuovi rischi, nuove paure. Bhopal, Cernobyl, Fukushima, in Italia le contaminazioni di diossina a Seveso, di amianto a Casale Monferrato sono disastri completamente antropici, legati all'uso incontrollato della tecnologia. Ma antropiche sono anche le alluvioni provocate dall'imbrigliamento dei fiumi tra ali di cemento, le frane provocate da urbanizzazioni selvagge. Da questo punto di vista, la categoria di “antropocene", l'idea che l'azione umana abbia alterato profondamente $\mathrm{i}$ cicli della natura contro la sopravvivenza stessa della vita in ogni sua dimensione sul pianeta è certamente adeguata a descrivere l'infittirsi delle catastrofi antropiche. Come pure, l'idea che alcune acquisizioni scientifiche possano poi prendere strade impreviste, portando a nuove catastrofi o peggiorando la vulnerabilità delle popolazioni, quelle che sono state definite "trappole del progresso". Utile è pure la visione globale insita nella categoria di antropocene: gli impatti globali dello scambio, le interrelazioni che legano i fenomeni a livello mondiale, così evidenti nel caso di Cernobyl e ancora di più nell'attuale pandemia, ma presenti anche a livelli meno visibili. Si pensi agli interventi umanitari dopo le grandi catastrofi e all'esportazione di modelli di vita e di insediamenti abitativi. Il rischio, mi pare, potrebbe essere quello di fare un uso approssimativo e ideologico del concetto: l'umanità che scivola verso la sua distruzione, quasi un ritorno del catastrofismo apocalittico. Senza perdere di vista gli intrecci globali, rimane importante contestualizzare gli eventi catastrofici: studiare le diverse vulnerabilità, i rapporti politici e sociali, le dinamiche di breve e di lungo periodo, le differenze geografiche e istituzionali e infine fare riemergere le esperienze e le visioni dei soggetti coinvolti.

Puoi evidenziare le ragioni per cui nel tuo testo accosti le memorie di esperienze traumatiche prodotte dalla Seconda guerra mondiale a quelle di eventi catastrofici come alcuni recenti terremoti? In che cosa l'esperienza della popolazione di Amburgo, che vive il trauma dei bombardamenti nel 1943, ̀̀ accomunabile a quella della popolazione di Laviano, in Irpinia, che si vede spazzare via il paese in occasione del terremoto del 1980? 
Come ho scritto nel libro, guerra e catastrofi hanno alcuni elementi in comune insieme a differenze cruciali. La grandezza, l'estensione territoriale, il coinvolgimento di milioni e milioni di persone, la profondità storica fanno della guerra un evento incommensurabile e incomparabile. La Seconda guerra mondiale divide il Novecento e riscrive la mappa geopolitica dell'Europa e del mondo, innescando dinamiche di lungo periodo e processi di rammemorazione complessi, spesso conflittuali. La sua memoria si divide inoltre lungo faglie politiche ed etiche, ed è più che mai soggetta alla manipolazione della sfera pubblica. C'è inoltre un elemento decisivo che caratterizza la violenza di guerra e in generale la violenza politica, ed è l'intenzionalità e la brutalità con cui i perpetratori danno la morte e umiliano la vittima, provocando una ferita quasi non rimarginabile nei sopravvissuti. La catastrofe può produrre un grande numero di vittime e distruggere le fondamenta sociali di una comunità, ma ha ovviamente implicazioni etiche e politiche meno evidenti. Una grande catastrofe può comunque provocare trasformazioni istituzionali di notevole rilevanza, far cadere governi, indurre cambiamenti di regime politico, innescare o interrompere processi di sviluppo economico.

Entrambi gli eventi mettono in moto processi di ricostruzione che si legano nelle varie fasi storiche alle tecniche architettoniche e alle idee dominanti sugli spazi urbani, come ad esempio quelle della modernizzazione e dello sviluppo che hanno caratterizzato molte delle ricostruzioni nel Novecento.

Entrambi mettono in moto processi di elaborazione del ricordo: il ricordo del passato mitico, le rovine, le vittime... Quale rapporto si crea con questo passato? Le comunità distrutte dalle bombe o dai terremoti devono decidere se cancellare le rovine o provare a ripristinare il paesaggio perduto. Ma ci sono anche coloro che sono stati delocalizzati. Quale il loro inserimento nel luogo nuovo, quale il rapporto con il paese abbandonato?

Anche in questo caso bisogna sottolineare la differenza tra la delocalizzazione di un villaggio abbattuto da un terremoto, e la delocalizzazione forzata o la deportazione di intere popolazioni da un'area geografica a un'altra attraverso nuovi confini.

Si tratta di differenze cruciali. Ma, nello stesso tempo, possiamo rilevare alcune dinamiche, alcuni processi di rammemorazione simili: la dimensio- 
ne del lutto e la sua trasmissione attraverso le generazioni, la pluralità dei discorsi e delle narrazioni che hanno riconfigurato gli spazi geografici e politici. Nei racconti di catastrofi appare sempre l'eco della guerra. Chi ha vissuto l'esperienza di un terremoto pensa a un bombardamento improvviso e devastante, e paragona le macerie a quelle provocate dai raid aerei.

[...] stava correndo come una pazza, tutta bagnata dalle sue lacrime. Lei non sapeva dove era. [...] Tutto è crollato, tutto è crollato, tutto!

È il racconto di una donna che vaga tra le macerie del terremoto che devastò Mexico City nel 1985. In un attimo si perde tutto: persone, affetti, oggetti che hanno accompagnato la vita, segnato l'identità. La perdita della casa spezza la continuità delle storie individuali e crea spaesamento. Lo spaesamento è poi aggravato dalla perdita del "luogo amato": lo spazio della vita, parte integrante dell'identità delle persone, la cui scomparsa può provocare quella "crisi della presenza" di demartiniana memoria.

Ecco ancora alcuni brani significativi tratti dal mio volume: a raccontare sono una donna di Laviano, paese campano distrutto dal terremoto del 1980, e alcuni testimoni di Longarone cancellato dal disastro della diga del Vajont.

L'unica cosa che non era caduta era il castello, questo tritone: questa vasca che c'è in piazza, questa chiesetta qui che adesso è stata ristrutturata dalla soprintendenza delle belle arti e poi un palazzo proprio all'inizio del paese e un altro palazzo [...] questi erano gli edifici che erano rimasti in piedi, il resto era tutto... ma la cosa strana dici: ma dov'è, dove sono tutte quelle case, sembravano un mucchietto di pietre, dici, ma dove sono finiti i mobili di queste case, può essere?! [...]. Mo la parte alta del paese, il castello, dove c'erano tante case una sull'altra, erano tantissime, era un mucchietto di pietre [...] veramente era un mucchietto di polvere... dove erano finiti i mobili!? [...] Non c'era più niente, era desolato, era come se gli spazi si fossero ristretti, sembrava tutto piccolino... è vero? La piazza piccolina, sembrava che tu dalla piazza nu momento arrivavi qua sopra, mentre prima dovevi camminare un bel po' per arrivare qua... come se si fosse ristretto il paese...

Al mattino ho rivolto lo sguardo a Longarone e ho visto questa riga diritta, questa piana bianca [...] sono rimasto così, non mi rendevo conto. 
Cosa avrei dovuto vedere? So quello che ho visto e non ricordo cosa c'era al suo posto il giorno prima [...] c'è voluto del tempo per ricostruire l'immagine normale che avevo da quel posto [...] Vedere Longarone il 9 e il 10 ottobre $[\ldots]$ non riuscivo a fare gli accostamenti $[\ldots]$ è stato uno choc incredibile.

Non c'erano più contorni, non si distingueva più niente; una cosa strana come se fosse vuoto sotto

Longarone era un ghiaione, ho guardato il paese e non c'erano più ponti né niente...

Quasi identico è il racconto di Nossack, che scrive dopo il terribile bombardamento di Amburgo del 1943:

Immaginate di chiudere gli occhi per qualche secondo, e quando li riaprite, non c'è più nulla di quello che c'era prima.

Il bombardamento appare come un lampo che squarcia lo scorrere del tempo, una catastrofe che si abbatte inaspettata su una natura immota e inconsapevole. L'istante prima è la natura dolce dell'estate nordica ignara della guerra.

La brughiera stava appena cominciando a fiorire. Sul ciglio delle strade c'erano piccoli ciuffi di campanelle.

Dopo, il nulla. I riferimenti concreti di una vita si erano persi sotto le macerie:

Non c'era rimasto nulla, nemmeno il più piccolo ninnolo, di tutte le cose che avevamo amato e ci erano appartenute. Se fosse rimasto qualcosa, non avremmo smesso di accarezzarlo, perché sarebbe stato come se portasse dentro di sé l'essenza di tutti gli altri oggetti.

Quel che vedevamo intorno non ci ricordava in alcun modo ciò che era andato perduto. ... Era qualcosa di diverso, era l'estraneità in sé, era l'autentico non-possibile. [...] Dove un tempo lo sguardo incontrava le mura delle case, adesso una muta pianura si stendeva verso l'infinito.... [...] una pianura di macerie... non c'erano vie traverse che portassero nel groviglio... le cose erano arruffate l'una nell'altra... ma ogni cosa stava in assoluto silenzio, immobile, immutabile, spogliata del tempo, era divenuta eterna. 
Il paesaggio è perduto per sempre. E la ricostruzione si sovrappone poi alle rovine e muta ancora più indelebilmente il paesaggio. E capitato ai paesi travolti dalla guerra, è capitato ai paesi distrutti da terremoti o alluvioni.

Dal libro emergono considerazioni che fanno pensare alle catastrofi come a "grandi occasioni" che possono favorire accelerazioni o modificare radicalmente equilibri politici, rapporti di potere, forme di governo. Si potrebbe allora sostenere che le modalità e le forme delle ripartenze siano determinate in prima istanza dalla "interpretazione" di una catastrofe, le attribuzioni di responsabilità e la mobilitazione dei diversi attori/soggetti collettivi intenzionati ad orientare la ripartenza secondo i rispettivi interessi? A tuo avviso, situazioni di ripartenza possono dare luogo a scenari politici e sociali radicalmente nuovi rispetto allo scenario precedente una catastrofe?

I disastri sono "esperienze sociali ricorrenti e agenti di cambiamento che segnano l'intersezione fra natura e cultura” (Oliver-Smith 1999), e rappresentano un turning point nella vita pubblica e nelle vite private. Sono, hanno scritto Olson e Gawronski (2001), come una biopsia, un taglio nella società che ne rivela politica, economia e salute sociale; costituiscono dei detonatori di problemi e conflitti preesistenti.

Possono provocare, in determinate congiunture, accelerazioni fatali che determinano mutamenti politici e socio-economici epocali: il terremoto di Managua del 1972 causò la caduta del dittatore Somoza in Nicaragua e quello del Messico del 1985 portò alla crisi del sistema monopartitico, autoritario e illiberale, che dominava il paese da molti anni, aprendo un processo di transizione verso un sistema multipartitico e liberale. Il ciclone tropicale che colpì l'area orientale del Pakistan nel 1970, provocando la morte di circa 500.000 persone, causò l'insurrezione nazionale e la guerra civile che condusse alla formazione dello stato indipendente del Bangladesh.

In Gujarat il terremoto del 2001 è stato il pretesto per demonizzare ed emarginare la minoranza musulmana, introducendo un antagonismo religioso nelle dinamiche della ricostruzione. Il partito nazionalista indù ha usato il disastro per rafforzare ed estendere le sue basi: oltre a controllare le risorse per la ricostruzione, ha combattuto le pratiche religiose sincretiche, abbattuto vecchi templi legati a sette religiose locali, manipolato la tradizione culturale promuovendo, anche attraverso 
l'edificazione di imponenti monumenti, figure storiche appartenenti all'ideologia integralista indù in contrasto con la memoria del mahatma Gandhi, simbolo della convivenza tra etnie e religioni diverse (Simpson 2013).

La catastrofe può accelerare processi di cambiamento già in atto, come nelle fasi di transizione, verso obiettivi "modernizzanti". Il terremoto del 1966 in Uzbekistan fu l'occasione per il regime comunista di varare una ricostruzione radicale di Tashkent, facendone "la città modello della modernità sovietica nelle periferie arretrate. La modernizzazione richiese la costruzione di strade ampie, piazze e nuovi edifici, e ovviamente il sacrificio di case tradizionali e di interi quartieri"(Buttino 2015). Lo spazio urbano venne completamente ridisegnato, e cancellato per sempre quel labirinto di viuzze che univano case, botteghe dei bazar, moschee, karavanseij che si possono ancora vedere nelle mappe ottocentesche.

In Italia, in particolare nel Mezzogiorno, i terremoti sono stati considerati l'occasione per accelerare processi di modernizzazione o di "razionalizzazione" del tessuto urbano, come nei casi di Messina e della valle del Belice descritti da Giacomo Parrinello (2015).

Parrinello ha analizzato la lunga storia della ricostruzione di Messina, mostrando come le scelte urbanistiche e le dinamiche della ripartenza abbiano influenzato profondamente il futuro della città, trasformandola da importante centro portuale, punto di riferimento di traffici mondiali, in una città caratterizzata dalla predominanza del settore edilizio e del settore terziario. Il caso, che qui sarebbe troppo lungo da riproporre, è interessante proprio perché l'autore analizza la ricaduta delle scelte sul lungo periodo, gli attori sociali che via via intervengono nel negoziare le soluzioni e i fattori che agiscono sulla strada a mutare gli esiti previsti. Identico approccio Parrinello ha applicato allo studio del Belice, dove, all'indomani del terremoto del 1968, era stato varato un progetto di pianificazione del territorio (la città-territorio) un vero e proprio intervento di ingegneria sociale che avrebbe dovuto portare la valle verso la modernità e il benessere. Il progetto prevedeva la costruzione di nuovi villaggi a fianco di quelli distrutti e una serie di strade e infrastrutture che avrebbero dovuto attirare le imprese e produrre sviluppo. Il progetto, ispirato alla cultura architettonica e urbanistica del tempo, era interamente calato dall'alto e non teneva in alcun conto 
le esigenze materiali e culturali delle popolazioni. Eppure, proprio gli abitanti dei comuni della valle del Belice avrebbero potuto intervenire e dialogare con gli esperti sull'organizzazione del proprio territorio e del proprio futuro, perché da molti anni lottavano per lo sviluppo della valle, chiedendo la costruzione di una diga che portasse l'acqua per le coltivazioni, ed erano organizzati in comitati che avrebbero potuto costituire degli interlocutori privilegiati delle istituzioni preposte alla ricostruzione e che invece vennero disconosciuti e repressi. Il piano di sviluppo proposto fallì completamente: nessuna impresa arrivò nella valle del Belice, e le infrastrutture costruite per ospitare lo sviluppo industriale sorsero imponenti intorno al vuoto. Altri problemi e disagi si verificarono per la ricostruzione dei vecchi abitati: il caso più noto è quello di Gibellina, il cui vecchio centro diruto è stato coperto dal famoso cretto di Burri, una sorta di sudario disteso sulle rovine, mentre il nuovo paese è stato ricostruito a molti chilometri di distanza dal vecchio paese, con un'architettura del tutto estranea alla cultura dei luoghi, mai interamente accettata dagli abitanti. Il dato interessante, che potrebbe insegnare molto sulle ripartenze e le ricostruzioni, è che la valle del Belice, poi, si è sviluppata: da latifondo cerealicolo si è trasformata in una valle di uliveti e vigneti, avviando finalmente lo sviluppo sognato. E questo è avvenuto con la costruzione di quella diga che la popolazione negli anni Cinquanta e Sessanta, ben prima del terremoto, aveva individuato come la soluzione per l'economia della valle, e per cui aveva lottato costituendo quei comitati che invece i pianificatori del post-terremoto non avevano voluto ascoltare.

Negli ultimi decenni studi storiografici, antropologici e di psicologia sociale hanno dedicato notevole interesse alle esperienze traumatiche determinate da grandi catastrofi, alle modalità di elaborazione e ai processi di sedimentazione $o$, al contrario, di rivisitazione critica che ne sono seguiti nel tempo. La ricerca sui traumi si è spesso intrecciata con il crescente interesse, almeno a partire dagli anni Novanta del Novecento, per le figure dei testimoni (l'era dei testimoni tematizzata da A. Wieviorka), tendenzialmente fatti coincidere con le vittime delle grandi catastrofi novecentesche. Le ragioni di tale attenzione sono molteplici, in parte evidenti e in parte più complesse, dati anche i nessi con pratiche di politiche della memoria diversamente promosse da numerosi paesi europei soprattutto dopo il 1989. Come spieghi il minor interesse per strategie 
e modalità di superamento dei traumi, di ripresa della "vita quotidiana", e di ripartenza atte a riconfigurare paesaggi, città, relazioni sociali e interpersonali portate via dalle catastrofi?

In Italia, come è stato detto ampiamente, prevale la cultura dell'emergenza a livello centrale come a livello locale. Alluvioni, frane, terremoti ed eruzioni vulcaniche hanno colpito l'Italia da millenni e la colpiranno ancora in futuro. Il nostro paese vive di fatto di emergenza, non di prevenzione, e sull'emergenza è cresciuto un vero e proprio "professionismo dell'emergenza" (Guidoboni e Valensise 2013). Come hanno scritto Boschi e Bordieri (1998),

Dal punto di vista elettorale, del controllo del consenso, della gestione delle clientele, la prevenzione del terremoto non rende. Anzi. È un affare in perdita. Imporre agli elettori un costo maggiore nella progettazione e nella costruzione degli edifici e delle opere pubbliche per renderli antisismici senza un motivo immediatamente evidente e per evitare un rischio apparentemente remoto, o addirittura vietare di costruirli, può alienare molte simpatie elettorali. [....] è invece altamente redditizio elargire aiuti e risarcire danni provocati da un'imprevedibile "calamità naturale", sotto la spinta dell'emergenza e della necessità immediata di dare un "tetto ai terremotati".

Concentrarsi sull'emergenza significa poi trascurare l'analisi delle vulnerabilità e dei criteri di prevenzione e mitigazione, e, infine, non tenere conto delle esperienze passate. Mancano studi e confronti fra i vari casi, manca in effetti una cultura della valutazione che misuri le decisioni prese all'inizio, i tempi lunghi della ricostruzione e i risultati raggiunti. Capita di perdere il nesso fra il nuovo habitat e la catastrofe. Proprio rispondendo a queste domande, ho pensato al caso di Napoli, città in cui vivo, la cui geografia urbana è stata profondamente trasformata dalle costruzioni dovute alla legge 219 del post-terremoto, con esiti a volte disastrosi. Lo sguardo sfiduciato si ferma sui risultati distorti, sui grandi quartieri ghetto sorti nelle periferie in cui domina la criminalità organizzata, saltando il lungo cammino che li ha prodotti e resi tali. Ci si dimentica la loro origine. Studiare le decisioni iniziali, confrontarle con i risultati, ripercorrere i vari momenti di svolta, fare riemergere gli attori sociali intervenuti in varie forme potrebbe invece fornire informazioni importanti per evitare errori nelle future "ripartenze". 
C'è poi il ruolo della memoria. Nei casi in cui la catastrofe ha distrutto intere comunità e mietuto molte vittime, la necessità di superare il trauma può dar luogo alla sua rimozione. L'attaccamento ai luoghi, il desiderio di restare nella propria casa può portare all'oblio e alla sottovalutazione del rischio. In alcuni casi, le istituzioni che non sono riuscite a prevenire e combattere un disastro possono esse stesse intenzionalmente oscurare l'evento. Come ad esempio accadde a San Francisco nel 1906, dove il governatore, per oscurare la pericolosità sismica della città che avrebbe ostacolato il turismo, oscurò il terremoto dando la colpa delle distruzioni all'incendio che si propagò a causa dell'entità della scossa: il disastro si doveva chiamare "il grande incendio" e non "il grande terremoto", una rappresentazione che si sarebbe diffusa nei media e protratta nel tempo (Svensen 2010). Salvatore Botta (2013) ha mostrato quanto sia stato difficile per lo Stato unitario italiano, disarmato di fronte alle caratteristiche morfologiche delle regioni acquisite al nuovo regno, affrontare i terremoti e mappare i territori a rischio. Molti dei comuni classificati a rischio sismico protestavano contro le regole edilizie troppo stringenti e facevano di tutto per uscire dalla classificazione. Significativa è, da questo punto di vista, la vicenda di Casamicciola, nell'isola di Ischia, già inserita nei casi a maggior rischio sismico dai Borboni, i quali avevano emanato un editto che vi proibiva la costruzione di fabbricati. Le regole non erano poi state rispettate, e ciò aveva provocato i disastri del 1828, del 1881 e del 1883, in cui morirono 2.333 persone. Anche allora, le autorità locali combatterono per nascondere l'accaduto e non rientrare nella mappa dei maggiori rischi sismici, per non ostacolare il flusso turistico. Il 21 agosto del 2017 un terremoto di bassa entità ha di nuovo colpito Casamicciola nella stessa area del 1883, e si è scoperto che si erano costruite case senza alcun criterio antisismico. Eppure a Napoli, per indicare un incidente, una sciagura, si usa il detto: "è successo Casamicciola”! Il linguaggio, come spesso succede, si è probabilmente staccato dal suo riferimento reale.

L'oblio istituzionale può rafforzare il desiderio delle comunità colpite di dimenticare il trauma. E questo in qualche misura potrebbe anche influenzare le ricostruzioni storiche.

Resta il fatto che, come ho detto nella risposta alla prima domanda, gli storici, con poche eccezioni, non si sono misurati sul tema. 
Ripartenze e spaesamenti: i tuoi studi sulle memorie di traumi provocati da terremoti mettono in evidenza le numerose difficoltà che le comunità sono costrette ad affrontare per risollevarsi dalle macerie. Di fronte a eventi che distruggono o deturpano in maniera insanabile gli spazi della vita e i paesaggi familiari, anche le più sensibili ricostruzioni non riescono a prevenire sentimenti di "spaesamento", di non riconoscimento del proprio paese e del paesaggio perduto. Anche là dove si è cercato di ricostruire il distrutto "dov'era com'era”, come nel caso di San Michele del Serino, lo spaesamento rimane (249). La comparazione tra casi di Gibellina (Sicilia) - edificata ex novo a 30 chilometri dal paese scomparso secondo un "creativo" progetto architettonico - e di Conza (Campania), ricostruita nel rispetto della volontà della comunità locale, ci offre utili indicazioni su pratiche più o meno virtuose di ricostruzione. Dalle memorie indagate possiamo trarre indicazioni di principio per orientare processi di ripartenza?

A me sembra che il problema sia spesso l'incapacità da parte di chi interviene - governo o istituzione internazionale - di dialogare con la popolazione locale e di tenere conto delle culture e delle risorse preesistenti. Come mostrano il caso del Belice e anche quello irpino non si innesta uno sviluppo industriale se non lo si lega alle caratteristiche del territorio. Non è un caso che il decollo dell'economia del Belice sia nato dall'agricoltura con l'impulso degli stessi abitanti, e che nei territori del cratere, in Campania e in Basilicata, abbiano attecchito le industrie agroalimentari che hanno potuto inserirsi più organicamente nel sistema economico locale.

Le catastrofi provocano distruzioni e vittime nei territori più vulnerabili, che coincidono quasi sempre con i paesi del Sud del mondo, cui viene applicato lo stigma dell'arretratezza, come è avvenuto anche in Italia nei terremoti che hanno colpito ripetutamente il Mezzogiorno: l'arretratezza diventa in questi casi la causa prima della catastrofe. Le popolazioni colpite sono condannate per la loro mancanza di adeguate conoscenze e preparazione, e devono essere indirizzate dall'alto verso la modernità e il progresso con un'opera di ingegneria materiale e sociale. Come hanno mostrato molti fra gli studiosi che hanno analizzato catastrofi e ricostruzioni nei paesi non occidentali, c'è in genere un'attenzione quasi nulla alle costruzioni "vernacolari”, vissute come espressione di povertà e arretratezza non solo dalle agenzie governative o non 
governative, ma anche dai community leader che sono spesso coloro che dialogano con gli interlocutori esterni.

Esemplare è il caso dei villaggi costieri dell'India distrutti dallo tsunami del 2004 studiati da Jennifer Duyne Barenstein (2021). Le comunità costiere della regione del Tamil Nadu avevano una forte cultura abitativa e una particolare capacità edificatoria. Molte case vernacolari, demolite e sostituite con case presumibilmente resistenti ai rischi multipli, erano di buona qualità, ecologicamente sostenibili, economiche, belle e più confortevoli delle case costruite dalle agenzie esterne. Le case locali erano state costruite in funzione dell'attività prevalente della pesca, ed erano proiettate verso l'esterno con verande e alberi in cui si svolgeva parte delle attività quotidiane della famiglia e si sviluppava la socialità tra vicini. Erano state costruite seguendo rituali e indicazioni sacre, e le donne avevano avuto un ruolo preminente nel seguire i lavori. Nell'intervento post-tsunami, il suolo è stato completamente spianato per edificare le nuove moderne case a schiera, che sono sorte in una specie di deserto, senza verande, senza alberi, escludendo le donne dalla partecipazione alla loro progettazione ed eliminando quello spazio fondamentale per la vita quotidiana della comunità intorno alle abitazioni. L'impatto della ricostruzione post-tsunami sul patrimonio naturale e architettonico dell'India, scrive Duyne, indica che le risposte delle agenzie esterne al disastro costituiscono una seria minaccia per il benessere delle comunità, e che le conseguenze dei programmi di ricostruzione socio-culturale insensibili alle culture locali possono essere più devastanti del disastro stesso. Cancellando i tradizionali habitat costieri attraverso la demolizione di case intatte e l'abbattimento di migliaia di alberi, le agenzie esterne non solo hanno privato le persone colpite della loro identità culturale e di un habitat vitale per il loro benessere, ma hanno anche minato il potenziale di sviluppo turistico di una fascia costiera straordinariamente bella.

In questo caso siamo di fronte a una finta modernizzazione: le case a schiera hanno rinchiuso le famiglie negli interni, peggiorando la condizione di tutti ma in particolar modo di donne e bambini.

Come abbiamo visto nel caso del Belice, anche in Italia i terremoti sono stati l'occasione per far partire processi di modernizzazione non sempre integrati con il territorio. Il caso del post-terremoto campano e lucano presenta invece una maggiore complessità: vi troviamo un dialogo più 
stretto fra i comuni e il centro, la mobilitazione attiva dei sindaci nella prima emergenza e il ruolo positivo che la neonata protezione civile affidò loro, e infine la scelta autonoma dei comuni rispetto al modello della ricostruzione. Gabriele Moscaritolo (1999) ha confrontato i due modelli di ricostruzione: il dov'era com'era di Sant'Angelo dei Lombardi e la new town di Conza della Campania. E quello che emerge da questi studi è la partecipazione della popolazione alle decisioni, il ruolo delle giunte e dei consigli comunali nel prefigurare e organizzare la nuova configurazione del territorio. A Sant'Angelo dei Lombardi una felice congiuntura vide lavorare insieme una sindaca particolarmente combattiva e un architetto colto e preparato che dirigeva l'ufficio di piano della soprintendenza, posto proprio nella cittadina: già nel settembre del 1981 era pronto il piano di recupero, la cui supervisione era affidata alla soprintendenza, mentre i lavori per delega venivano affidati al comune. Non sempre e non tutto ha avuto esiti felici: lo spaesamento è continuato per molti che non si sono riconosciuti nelle ristrutturazioni o nei nuovi spazi, anche nelle situazioni migliori e più partecipate, e questo è parte di tutte le storie di traumi dove la perdita delle persone e dello spazio vitale è sovente irrecuperabile. Qui, come ha mostrato Moscaritolo, si instaura a volte un dialogo difficile fra le generazioni: chi è nato dopo il terremoto ed è cresciuto fra discorsi di lutto e di nostalgia, vorrebbe passare oltre. I nuovi spazi sono per lui, o per lei, gli unici spazi vissuti. Inoltre, alcuni paesi hanno vissuto conflitti laceranti, che si fanno sentire tuttora. Ad esempio a Laviano, paese letteralmente sbriciolato dalla scossa, ha prevalso il modello scelto dal sindaco del tempo contro l'opinione del comitato popolare: da paese arroccato sui poggi della montagna, si è trasformato in un conglomerato di condomini, un incolore quartiere urbano. L'autonomia, in questo caso, fu utilizzata dal sindaco per monopolizzare la ricostruzione, affidando il 90\% dei lavori a progettisti a lui direttamente legati (Ventura 2020). Tutto ciò ha provocato, nella comunità, una ferita mai rimarginata. Una volta eletto, molti anni dopo, sindaco del paese, Rocco Falivena - che nell'Ottanta aveva guidato il comitato di lotta che si era opposto alle scelte di edificazione di allora - ha fatto costruire il plastico dell'antico paese, inaugurato il 20 novembre del 2010. Queste le parole del sindaco al momento dell'inaugurazione: "In occasione del trentennale abbiamo voluto privilegiare la memoria e far conoscere alle giovani 
generazioni (il 50\% della popolazione) il vecchio paese, un paese di cui non hanno memoria. Il nostro obiettivo è anche quello di riflettere sull'assetto urbanistico; la nuova città, quella ricostruita dopo il sisma, infatti, è totalmente estranea alla nostra cultura". Il plastico era un modo per sanare le ferite della perdita del luogo amato.

Il terremoto del 1980 ha colpito un territorio vastissimo e un numero enorme di comunità di varie dimensioni e caratteristiche, con storie ed esiti diversi. Proprio per questo, potrebbe rappresentare un vero e proprio laboratorio per studiare i processi di ricostruzione messi in opera, $\mathrm{i}$ motivi dei successi e quelli degli insuccessi.

Tra gli insuccessi c'è, ad esempio, la costruzione dei quartieri di edilizia popolare destinati ai terremotati nella periferia di Napoli e nel territorio metropolitano circostante. L'operazione fu inserita nel Piano per le Periferie di Napoli del post terremoto 23 novembre 1980, un progetto che riproponeva, ampliandola, la pianificazione che era stata fatta l'anno precedente per dare casa a famiglie senza tetto o abitanti in situazioni di estremo degrado, e allo stesso tempo migliorare la qualità delle abitazioni nelle periferie con un'opera complementare di ristrutturazione dell'abitato preesistente (Corona 2007). Il piano fu allora giudicato un progetto avanzato e innovativo da importanti urbanisti del tempo, ma i risultati, per quel che riguarda i quartieri di nuova costruzione, sono stati a dir poco deludenti.

Il terremoto a Napoli ha prodotto un numero minore di vittime, ma ha provocato danni enormi al patrimonio abitativo: quasi 10.000 edifici sono stati dichiarati inagibili e 112.000 abitanti hanno dovuto abbandonare le proprie case. Questa vasta popolazione di senzatetto-terremotati, proveniente soprattutto dai quartieri storici, viene distribuita in 50 campi container, negli alberghi e negli alloggi requisiti nelle aree a nord di Napoli, e poi riallocata in nuovi rioni con la promessa di una nuova condizione di "civiltà urbana". Nell'area metropolitana, i nuovi quartieri si pongono anche fisicamente al di fuori e contrapposti ai vecchi borghi, con la cui popolazione non si integreranno mai. In città, con l'idea di risparmiare suolo per poter creare parchi verdi e servizi, si sono costruiti grandi palazzi ispirati al modello Le Corbusier, che già aveva influenzato l'edificazione delle famose Vele di Scampia, costruite poco prima. Questi abnormi complessi si sono velocemente degradati e, attraverso intimidazioni e occupazioni abusive, sono diventati do- 
minio della camorra. Nel frattempo, i parchi verdi o non sono sorti o, quando sono sorti, non sono stati mantenuti, e le attrezzature sono state vandalizzate o abbandonate. Nei nuovi quartieri era stata concentrata la popolazione più fragile e precaria, e l'organizzazione dello spazio, i modelli abitativi, la concentrazione del disagio sociale in quartieri ghettizzati hanno prodotto risultati perversi, oggi difficilmente riparabili. Ilaria Vitellio, nel presentare un saggio sulla ricostruzione a Napoli e nella sua area metropolitana (2013), ha riportato le parole di Andrea Geremicca, assessore della giunta di sinistra del sindaco Valenzi dal 1975 al 1983:

Se oggi, trent'anni dopo, un urbanista dovesse indicare a uno studente tracce di quel 23 novembre del 1980 nell'area metropolitana di Napoli, dovrebbe prenotare un viaggio nel post-terremoto delle vite degli altri. Quello che venne battezzato come "Piano Napoli", poi trasformato in legge 219. La catastrofe che doveva diventare occasione per colmare la fame abitativa di Napoli. E che invece creò le cattedrali del disagio cronicizzato. Ventimila alloggi, oltre centomila vani, che dopo tre decenni restano l'eredità più pesante. Autentiche bombe sociali piantate, come una cintura di kamikaze, tutte intorno ai bordi di Napoli e degli altri 17 Comuni che le facevano da via di fuga cementizia. Sono nati, allora, i mostri di oggi. Taverna del Ferro, il bronx di Napoli. Rione Salicelle, girone infernale di Afragola. Parco Verde, a Caivano, il quartiere con la più alta densità di malavita minorile. La 219 di Melito, succursale dello spaccio degli scissionisti a Scampia. [...] Che cosa non ha funzionato? Intanto non avevamo strumenti urbanistici definiti, oggi diremmo integrati, se non i Piani di edilizia di zona, che ci dicevano già in quali aree mettere le mani. Poi non avremmo dovuto aggregare solo i ceti più poveri ed emarginati. Dovevamo accompagnare questa roba con un piano di sviluppo economico, invece volemmo il maledetto e subito!

Il caso riportato di ricostruzione post-terremoto in una regione indiana del Gujarat (2001) per opera di volenterose ONG determinate a "cogliere l'occasione" della ripartenza per intervenire sul tessuto sociale di quella comunità, cercando cosi di decostruire attraverso l'architettura e la riconfigurazione degli spazi consolidate gerarchie di casta e relazioni di genere, relativizza per certi verso l'effetto spartiacque delle catastrofi. Eventi catastrofici segnano inesorabilmente cesure indelebili tra un prima e un dopo, ma in fase di ripartenza il riferimento al prima e la volontà di recuperare il perduto sembrano prevalere. 
Nelle ripartenze sembra insomma crearsi un duro campo di tensione tra l'inevitabile necessità di proiettarsi nel futuro per creare un nuovo tessuto sociale di vita e l'altrettanto inevitabile, così appare, attitudine a riprendere e ricostruire il prima. È corretto pensare che i "prima" continuino a orientare in maniera quasi inconsapevole $i$ "dopo" che seguono ogni catastrofe?

Come ho detto prima, è molto difficile importare modelli dall'alto, e non sempre ciò dà i risultati previsti. Bisognerebbe accettare quel campo di tensione fra prima e dopo, riuscire a fare dialogare le generazioni, cercare di recuperare gli aspetti positivi di resilienza e anche di possibili culture del rischio fra le popolazioni, valutare correttamente le vulnerabilità. Il "perduto" significa lo spazio della vita, l’identità individuale e familiare.

I sopravvissuti di una catastrofe devastante esprimono quasi sempre il bisogno di rimanere o ritornare al luogo natio devastato. Il luogo distrutto resta un riferimento identitario fondamentale per gli abitanti, una sorta di ancoraggio contro la perdita, lo spaesamento, il lutto. I cittadini di Gibellina hanno vissuto come una violenza la copertura delle rovine del paese e, forse, si sarebbe dovuto rispettare i loro sentimenti.

Sono contraddizioni a volte insanabili, spesso divisive fra le generazioni. Inoltre è sempre difficile, se non impossibile, attuare azioni di ingegneria sociale, come insegna proprio il caso del Gujarat, dove un tentativo di modernizzazione opposto all'ideologia delle caste finisce, con il nuovo habitat, di isolare ancora di più le donne. Ma proprio a partire dalle donne, in alcuni casi che citerò fra poco, è stato possibile innovare e inaugurare un nuovo modello sociale e materiale. Il problema è sempre studiare le specificità di ogni contesto, riuscire a dare spazio alla mobilitazione dal basso, o, per lo meno, far dialogare i due livelli: locale e centrale.

Pensi che l'assunzione di una prospettiva di genere potrebbe arricchire, e come, la comprensione di processi di ripartenza in particolari situazioni di catastrofi? Per quanto riguarda la storia postbellica, vari studi hanno messo in luce il ruolo delle donne non solo nella raccolta delle macerie (le Trümmerfrauen in Germania), ma anche nella riorganizzazione della vita pubblica e politica, amministrativa e lavorativa. Cosa potrebbe, a tuo avviso, aggiungere una prospettiva di genere nell'analisi di situazioni di ripartenza dopo catastrofi "naturali"? 
In quasi tutti i casi studiati, in Italia come nel resto del mondo, sono le donne, che vivono male la costruzione di nuovi spazi anonimi e la perdita della socialità di prima, a essere più colpite dallo spaesamento. Se fossero state ascoltate prima? Si sarebbero potuti costruire habitat più umani? Tutte le ricerche sul tema affermano questo: le donne non vengono adeguatamente consultate nelle decisioni di rilocalizzazione, nel design delle nuove case, anche se la loro conoscenza e la loro esperienza nelle comunità, le loro idee basate sulla necessità e sulla pratica renderebbero molto più efficaci le decisioni.

Le donne hanno spesso, rispetto agli uomini, una maggiore conoscenza dei bisogni vitali. Molte ricerche hanno documentato il loro ruolo nell'assicurare, durante e dopo il disastro, cibo e rifugio (un ruolo storico assolto dalle donne durante la guerra), tuttavia sono in genere marginalizzate nelle decisioni da prendere sul da farsi. In molte comunità le donne prendono parte attiva alle iniziative relative ai disastri, sia nella leadership locale, sia nei ruoli di base; tuttavia, nell'organizzazione di pianificazione formale sono scarsamente rappresentate, e sono quasi sempre assenti nelle posizioni di formazione delle decisioni. Gli uomini controllano la maggior parte delle posizioni di leadership nei settori di intervento per la riduzione del rischio nelle agenzie governative, nelle organizzazioni accademiche e di aiuto. Il tipico modello di management del disastro è dominato da una cultura maschile.

Il ruolo delle donne nelle pratiche sociali, la loro capacità di sopravvivenza e adattamento, i loro network personali, la cura intima dei più vulnerabili e la conoscenza delle risorse del territorio sarebbero strumenti preziosi per organizzare l'emergenza come la ripartenza, nonché per migliorare il sistema di welfare di una società. Sono temi e riflessioni continuamente riproposti in tutti i rapporti scaturiti dalle riunioni internazionali che si sono occupate di gender e riduzione del rischio, con la raccomandazione di includere l'impiego delle donne in posizioni chiave nella gestione dell'emergenza e nell'organizzazione della ripartenza'.

International Gender Equality and Disaster Risk Reduction Workshop (Honolulu, agosto 2004); World Conference on Disaster Reduction (Kobe, 2005); Global Platform for Disaster Risk Reduction (Ginevra, 2007-UNISDR). Una discussione approfondita su tutte queste tematiche e sulle ricerche relative si trova in Enarson e Chakrabarti 2009. 
Sono raccomandazioni continuamente disattese dall'Italia, come rivela la gestione finora svolta nell'affrontare la pandemia del Covid-19: comitati tecnici, posizioni chiave nei ministeri e nelle istituzioni preposte alla mitigazione del rischio interamente maschili, e un welfare ancorato al lavoratore maschio, che proprio nei casi di crisi mostra la sua inadeguatezza ad alleviare le sofferenze economiche e sociali.

Gli studi mostrano poi come le donne risultino più vulnerabili ai rischi, oggi più di ieri. Soprattutto nei paesi del sud del mondo ci sono più donne in estrema povertà, più donne sole per l'emigrazione degli uomini verso le città, più madri sole e vedove. Nello tsunami che ha colpito Indonesia, India e Sri Lanka, l' $80 \%$ di vittime erano donne. In Italia la pandemia sembra produrre conseguenze economiche più gravi sulla popolazione femminile: sono in maggioranza le donne a perdere il lavoro.

Ovviamente anche in questo caso vanno studiati i contesti, considerate le differenze di genere preesistenti e quelle indotte da cattive politiche di recovery, e insieme occorre capire come integrare le donne nei ruoli di decisione, come rompere le resistenze maschili e agevolare i cambiamenti.

Accanto a ricerche che mettono in luce le difficoltà o i risultati negativi, come quella prima citata sui villaggi di pescatori del Tamil Nadu o quelle incentrate su paesi a elevato grado di dominanza maschile e segregazione delle donne, ci sono lavori che propongono casi di successo, mostrando le donne come agenti di cambiamento e di resilienza. Gruppi organizzati di donne riescono a gestire il management del disastro e a organizzare la ripartenza in Salvador, altri gruppi riescono a impostare le strategie relative alla ricostruzione materiale dell'habitat in Turchia a Marmara, e un importante ruolo di resilienza viene svolto dalle donne dopo la grande eruzione di Montserrat nel 1995: la loro spontanea preparazione si dimostra tanto importante da indurre i programmatori a inserirle formalmente nei piani di gestione del disastro (Enarson e Chakrabarti 2009).

Rispetto alle domande che stanno emergendo in considerazione delle enormi sfide ambientali cui siamo giunti, non pensi che una storiografia delle ricostruzioni, delle ripartenze e dei risollevamenti post-traumatici potrebbe offrire utili stimoli di riflessione e orientamento per il tempo presente? 
Non c'è dubbio che una storiografia delle ricostruzioni, ripartenze e risollevamenti potrebbe offrire stimoli importanti per il tempo presente, da moltissimi punti di vista. Innanzitutto, come si è visto nella precedente domanda, per il ruolo assegnato alle donne, che in Italia è pressoché nullo. La narrazione e l'interpretazione della pandemia sono affidate a esperti, medici ed epidemiologi, non sempre in accordo fra di loro e rigorosamente maschi (tranne rare eccezioni). Mai nelle quotidiane conferenze stampa televisive della protezione civile, che si svolgevano nel periodo del lockdown totale, abbiamo visto una studiosa donna commentare i numeri della pandemia, tra l'altro esibiti in maniera elementare, senza un'approfondita analisi statistica. Eppure sappiamo che esistono validissime ricercatrici in medicina come in statistica. Questa è stata un'occasione persa di ribaltare i ruoli di genere e di offrire alla società esempi positivi.

Inoltre, l'approccio alla catastrofe si esaurisce spesso in un discorso tecnocratico e scientifico. Una donna potrebbe forse accostare al discorso scientifico una visione di genere, umanamente più aperta alla dimensione privata dell'esistenza? Si tratterebbe di una dimensione fondamentale per organizzare il welfare del recovery e i sussidi.

Gli studi sulle catastrofi potrebbero poi insegnare molto sulla valutazione delle vulnerabilità e sulla loro mitigazione, nonché sulla costruzione di una cultura del rischio. L'analisi storica potrebbe fornire un grandissimo apporto allo studio degli effetti degli eventi catastrofici sul lungo periodo, per capire gli errori come gli esiti positivi, gli ostacoli incontrati, il modo di superarli...

Infine, la storia ha un ruolo imprescindibile nella trasmissione della memoria. E da questo punto di vista la storiografia, ci siamo accorti solo ora, ha fatto troppo poco. Ha dell'incredibile che una vicenda come quella della Spagnola, con milioni di morti, sia passata pressoché inosservata agli occhi degli storici. Non esiste quasi libro o capitolo di manuale sulla Prima guerra mondiale e sul dopoguerra che dedichi uno spazio pur minimo alla pandemia. Abbiamo scoperto in questi giorni che il piano per le epidemie in Italia non era aggiornato. L'oblio ostacola la prevenzione, impedisce la costruzione di una cultura del rischio, come abbiamo visto per i terremoti.

Nelle catastrofi si sviluppano narrazioni pubbliche che ripropongono stereotipi, esaltano alcuni aspetti degli eventi e ne celano altri. La storia 
può indagare il contesto in cui l'evento catastrofico si colloca, e contrastare racconti semplificati e convenzionali. Può inoltre far emergere le esperienze, le interpretazioni, le memorie soggettive, fondamentali per capire la dimensione complessa del disastro e i modi per superarlo.

\section{Letteratura citata nell'intervista}

Bankoff, Greg. 2002. Cultures of Disaster: Society and Natural Hazards in the Filippines. London-New York: Routledge.

Boschi, Ezio, e Franco Bordieri. 1998. Terremoti d'Italia. Il rischio sismico, l'allarme degli scienziati, l'indifferenza del potere. Milano: Dalai.

Botta, Salvatore. 2013. Politica e calamità. Il governo dell'emergenza naturale e sanitaria nell'Italia liberale (1861-1915). Soveria Mannelli: Rubbettino.

Buttino, Marco. 2015. Samarcanda. Storie di una città dal 1945 a oggi. Roma: Viella.

Corona, Gabriella. 2007. I ragazzi del piano. Napoli e le ragioni dell'ambientalismo urbano. Roma: Donzelli.

Duyne Barenstein, Jennifer. 2021. The Remembered Villages. The Impact of Post-Disaster Reconstruction on Communities' Housing Culture, Identity and Wellbeing in Tamil Nadu. In Il terremoto del 23 novembre 1980. Luoghi e memorie, a cura di Gabriella Gribaudi, Francesco Mastroberti, Francesco Senatore, 219-238. Napoli: Editoriale Scientifica.

Enarson, Elaine, e P.G. Dhar Chakrabarti, a cura di. 2009. Women, Gender and Disaster: Global Issues and Initiatives. New Delhi: SAGE Publications.

Gribaudi, Gabriella. 2005. Guerra totale. Tra bombe alleate e violenze naziste. Napoli e il fronte meridionale 1940-44. Torino: Bollati Boringhieri.

- 2020. La memoria, i traumi, la storia. La guerra e le catastrofi nel Novecento. Roma: Viella.

Guidoboni, Emanuela, e Valensise Gianluca. 2013. L'Italia dei disastri. Dati e riflessioni sull'impatto degli eventi naturali 1861-2013. Bologna: BUP.

Hewitt, Kenneth. 1997. Regions of Risk. A Geographical Introduction to Disasters. London-New York: Routledge.

Moscaritolo, Gabriele Ivo. 2020. Memorie dal cratere. Storia sociale del terremoto in Irpinia. Firenze: Editpess.

Oliver-Smith, Anthony. 1999. “'What is a Disaster?' Anthropological Perspective on a Persistent Question.” In The Angry Earth. Disaster in Anthropological Perspective, a cura di Anthony Oliver-Smith e Susanna M. Hoffman, 32-48. London-New York: Routledge.

Olson, Richard Stuart, e Vincent T. Gawronski. 2001. "Tapping Collective Memory of Disaster: Getting 'inside' the 1985 Mexico City Earthquakes.” International 
Journal of Mass Emergencies and Disasters 19, no. 3 (November): 108-18. https://doi. org/10.4000/rga.266.

Parrinello, Giacomo. 2015. Fault Lines. Earthquake and Urbanism in Modern Italy, New York-Oxford: Berghahn.

Pfister, Christian. 2011. “The Monster Swallows You': Disaster Memory and Risk Culture in Western Europe, 1500-2000". RCC [Rachel Carson Center for Environment and Societies] Perspectives 1: 3-23.

Simpson, Edward. 2013. The Political Biography of an Earthquake. Aftermath and Amnesia in Gujarat, India. London: Hurst.

Svensen, Henrik. 2010. Storia dei disastri naturali. La fine è vicina. Bologna: Odoya.

Ventura, Stefano. 2020. Storia di una ricostruzione. L'Irpinia dopo il terremoto. Soveria Mannelli: Rubbettino.

Vitellio, Ilaria. 2013. "Il territorio dell'oblio. La ricostruzione post-terremoto nell'area metropolitana di Napoli." In Terremoti. Storia, memorie, narrazioni, a cura di Gabriella Gribaudi e Anna Maria Zaccaria. Verona: Cierre (Memoria/Memorie 8).

Walter, François. 2009. Catastrofi. Una storia culturale. Vicenza: Colla (ed. or. 2008. Catastrophes. Une histoire culturelle XVI $-X X I^{e}$ siècle. Paris: Seuil). 